\title{
NUEVOS REGISTROS DE GÉNEROS Y ESPECIES DE ARAÑAS (ARANEAE) PARA LA HISPANIOLA, CON NUEVOS DATOS DE DISTRIBUCIÓN
}

\author{
Gabriel de los Santos ${ }^{1}$ y Giraldo Alayón ${ }^{2}$ \\ ${ }^{1}$ Museo Nacional de Historia Natural de Santo Domingo (MNHNSD). Calle César Nicolás Penson, \\ Plaza de la Cultura, Santo Domingo, República Dominicana. \\ g.delossantos@museohistorianatural.gov.do. \\ ${ }^{2}$ Museo Nacional de Historia Natural de Cuba (MNHNCu). Obispo 61, Plaza de Armas, Habana Vieja, \\ C. P. 10100, La Habana, Cuba. moffly@infomed.sld.cu
}

\section{RESUMEN}

Se registran por primera vez para la fauna de La Hispaniola las especies y géneros de arañas: Mimetus notius Chamberlin, 1923; Tapinopa bilineata Banks, 1893; Leucauge moerens (O. P.-Cambridge, 1896); Kapogea alayoi (Archer, 1958); Ctenus ottleyi (Petrunkevitch, 1930); Xysticus pellax O. P.-Cambridge, 1894; Megalostrata raptor (L. Koch, 1866); Mazax O. P.-Cambridge, 1898; Orchestina Simon, 1882; Neohannia Mello-Leitão, 1917; Pholcomma Thorell, 1869; Camillina Berland, 1919; Zelotes Gistel, 1848; y Tibellus Simon, 1875. También se presentan nuevas localidades para las especies endémicas: Ochyrocera cachote Hormiga, Álvarez-Padilla et Benjamin, 2007; Trujillina spinipes Bryant, 1948; Lausus pulchellus Bryant, 1948; Eustala bisetosa Bryant, 1948; Larinia minor Bryant, 1945 y el género Cobanus O. P.Cambridge, 1900. Se discuten brevemente algunos aspectos taxonómicos y biogeográficos de estas especies.

Palabras clave: Araneae, arañas, La Hispaniola, nuevo registro.

Title: New records of genera and species of spider (Araneae) from Hispaniola, with new distribution data.

\section{ABSTRACT}

New records of spiders genera and species of the Hispaniola fauna are included here. They are: Mimetus notius Chamberlin, 1923; Tapinopa bilineata Banks, 1893; Leucauge moerens (O. P.-Cambridge, 1896); Kapogea alayoi (Archer, 1958); Ctenus ottleyi (Petrunkevitch, 1930); Xysticus pellax O. P.-Cambridge, 1894; Megalostrata raptor (L. Koch, 1866); Mazax O. P.-Cambridge, 1898; Orchestina Simon, 1882; Neohannia Mello-Leitão, 1917; Pholcomma Thorell, 1869; Camillina Berland, 1919; Zelotes Gistel, 1848; y Tibellus Simon, 1875. Also, new localities for the endemic species Ochyrocera cachote Hormiga, Álvarez-Padilla et Benjamin, 2007; Trujillina spinipes Bryant, 1948; Lausus pulchellus Bryant, 1948; Eustala bisetosa Bryant, 1948; Larinia minor Bryant, 1945 and the genus Cobanus O. P.-Cambridge, 1900. are recorded. Some taxonomic and biogeographical aspects of these species are briefly discussed.

Key words: Araneae, spiders, Hispaniola, new record.

\section{INTRODUCCIÓN}

Sánchez-Ruiz (2009) resume de manera excepcional la historia sobre los estudios de la fauna actual de arañas en La Hispaniola (Haití y República Dominicana), desde finales del siglo XIX hasta el año 2007. Otro aporte importante lo hace Pérez-Gelabert (2008), recogiendo 322 especies actuales de arañas en su lista de artrópodos de La Hispaniola, basándose en la literatura publicada. 
Este número de 322 especies aumentaría rápidamente con cuatro nuevos registros publicados por Sánchez-Ruiz (2009), el nuevo registro del género Bolostromus Ausserer en la fauna actual de La Hispaniola por parte de Alayón y de los Santos (2009), así como la descripción de tres especies del género endémico Tainonia Huber (Huber y Astrin, 2009). Huber et al. (2010) continuaron sumando a la lista de arañas de la isla con la descripción de 22 especies del género Modisimus Simon, mientras que Sánchez-Ruiz et al. (2010) sumaron una nueva especie del nuevo género Cubanops Sánchez-Ruiz, Platnick et Dupérré. En el año 2011 se hacen dos nuevos registros de especies de la familia Araneidae (de los Santos y Carrero, 2011), así como la descripción de una especie de Wagneriana F. O. P.-Cambridge, 1904 (Alayón, 2011), endémica de la isla.

\section{OBJETIVO}

- Hacer nuevos registros de géneros y especies de arañas para La Hispaniola; dar a conocer nuevas localidades para cinco especies endémicas y el género Cobanus.

\section{MATERIALES Y MÉTODOS}

Se revisaron 1,494 especímenes procedentes de tres proyectos de investigación comprendidos durante el período 2009-2012. Además, también se examinó la colección del Instituto de Investigaciones Botánicas y Zoológicas (IIBZ) de la Universidad Autónoma de Santo Domingo y material adicional colectado por el segundo autor en varias visitas a la República Dominicana entre 1987-2009. El material fue examinado utilizando un microscopio estereoscópico Meiji Techno modelo EMZ-5TR, con aumento hasta 45X y otro marca Wild con aumento hasta 50X. Todos los ejemplares se encuentran depositados en las colecciones del Museo Nacional de Historia Natural de Santo Domingo (MNHNSD), el IIBZ y la colección personal del segundo autor (CGA).

\section{NOMENCLATURA}

$\mathrm{msnm}=$ metros sobre el nivel del mar.

mbnm $=$ metros bajo el nivel del mar.

psnm $=$ pies sobre el nivel del mar.

\section{RESULTADOS}

\section{NUEVOS REGISTROS PARA LA HISPANIOLA}

Familia Oonopidae

Orchestina Simon, 1882

Material examinado: 2 juv., MNHNSD 09.525. Boca de Yuma, San Rafael del Yuma, prov. La Altagracia, República Dominicana. Parque Nacional del Este. 26.III.2002, B. Farrel, K. Guerrero.

Familia Mimetidae

Mimetus notius Chamberlin, 1923

Material examinado: 1 , CGA. 3 km Valle de Elisa, prov. Monte Cristi, República Dominicana. 5.II.1991, G. Alayón. 
Familia Theridiidae

Pholcomma Thorell, 1869

Material examinado: 1 , IIBZ. Formon, Massif de la Hotte, Haití. 2-3.II.2006, R. Bastardo.

Familia Lyniphiidae

Tapinopa bilineata Banks, 1893

Material examinado: 1 , 1 juv., MNHNSD 09.1238. La Pirámide, Constanza, prov. La Vega, Parque Nacional Valle Nuevo, República Dominicana. UTM 19Q 331219m.E 2069060m.N, 2,388msnm. 17.I.2011, S. Carrero, G. de los Santos, sobre Danthonia domingensis Hackel \& Pilger. 2 q, 1 juv., MNHNSD 09.1239. La Pirámide, Constanza, prov. La Vega, Parque Nacional Valle Nuevo, República Dominicana. UTM 19Q 331219m.E 2069060m.N, 2,388msnm. 10.VI.2010, S. Carrero, G. de los Santos, sobre Danthonia domingensis. $10 \AA$, 1 juv., MNHNSD 09.1240. Sabana Quéliz, Constanza, prov. La Vega, Parque Nacional Valle Nuevo, República Dominicana. UTM 19Q 327724m.E 2074956m.N, 2,290msnm. 25.VIII.2010, S. Carrero, G. de los Santos, sobre Danthonia domingensis. 1q, 1§̋, MNNHSD 09.1236. La Pirámide, Constanza, prov. La Vega, Parque Nacional Valle Nuevo, República Dominicana. UTM 19Q 331313m.E 2069156m.N, 2,395msnm. 12.VI.2010, S. Carrero, G. de los Santos, sobre Danthonia domingensis. 2 juv. MNHNSD 09.1237. La Lechuguilla, Constanza, prov. La Vega, Parque Nacional Valle Nuevo, República Dominicana. UTM 19Q 331501m.E 2070693m.N, 2,412msnm. 26.VIII.2010, S. Carrero, G. de los Santos, sobre Danthonia domingensis.

Familia Tetragnathidae

Leucauge moerens (O. P.-Cambridge, 1896)

Material examinado: 1, CGA. Parque Nacional Armando Bermúdez, República Dominicana. 4.XII.2008, G. Alayón.

Familia Araneidae

Kapogea alayoi (Archer, 1958)

Material examinado: $1 \uparrow$ juv., CGA. Honduras - Matadero, Baní, prov. Peravia, República Dominicana. 17.XII. 2009, G. Alayón.

Familia Ctenidae

Ctenus ottleyi (Petrunkevitch, 1930)

Material examinado: 1q, CGA. El Cajuilito, El Carril, Bajos de Haina, San Cristóbal, República Dominicana. 12.VIII.1987, E. Marcano y L. F. de Armas (bajo piedras).

Familia Hahniidae

Neohannia Mello-Leitão, 1917

Material examinado: $q$ y juveniles, CGA. Parque Nacional Armando Bermúdez; República Dominicana. 4.XII.2008, G. Alayón.

Familia Corinnidae

Mazax O.P.-Cambridge, 1898

Material examinado: 1 , MNHNSD 09.1263. Aproximadamente $5 \mathrm{~km} \mathrm{~N}$ de Pedregal, Distrito Nacional, República Dominicana. $18^{\circ} 38.559^{\prime}$ N 70 03.506’W. 18.XI.2002, D. Pérez, R. Bastardo, B. Hierro. 19 MNHNSD 09.1228. Sendero Rabo de Gato, Puerto Escondido, Duvergé, prov. 
Independencia, República Dominicana. 18¹8'41.2”N 71³4’52.8”W, 434msnm. 09.IV.2009, R. Ortiz, G. de los Santos. 290 , MNHNSD 09.1227. Las Marías, Galvan, prov. Bahoruco, República Dominicana. 18²9'26.5'N 71²2’49.8'W, 22msnm. 04.IX.2009, G. de los Santos.

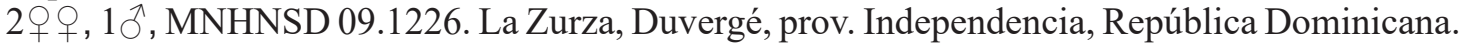
$18^{\circ} 24^{\prime} 25.1$ 'N 71³4'25.4"W, 28 mbnm. 05.VII.2009, G. de los Santos. 1 9, MNHNSD 09.1225. La Furnia, Las Clavellinas, Los Ríos, prov. Bahoruco, República Dominicana. 18³0’37.3'N 71³4'14.8"W, 22mbnm. 03.VII.2009, G. de los Santos. 1ð̋, MNHNSD 09.1224. Loma Los Cedros, Honduras, Baní, prov. Peravia, República Dominicana. UTM 19Q 351473m.N 2036495m.E, 656 msnm. 23.X.2009, G. de los Santos. 1, MNHNSD 09.1221. Las Barías, La Descubierta, prov. Independencia, República Dominicana. 18³3'43.8'N 7143'31.4"W, 21mbnm. 08.I.2010, G. de los Santos. $2{ }^{\circ}$, MNHNSD 09.1222. La Azufrada, La Descubierta, prov. Independencia, República Dominicana. 18³3'46.8'N $71^{\circ} 41^{\prime} 52.0$ "W, 18mbnm. 12.II.2010, G. de los Santos. 29 , , MNHNSD 09.1223. El Matadero, Honduras, Baní, prov. Peravia, República Dominicana. UTM 19Q 349857m.E 2035441m.N, 464msnm. 27.VI.2009, G. de los Santos. 1 , MNHNSD 09.427. El Matadero, Honduras, Baní, prov. Peravia, República Dominicana. 25-26.X.2008, V. De la Rosa, A. Hilario, G. de los Santos. 1q, MNHNSD 09.1262. En finca de aguacates, Puerto Escondido, Duvergé, prov. Independencia, República Dominicana. $18^{\circ} 20^{\prime} 54.8^{\prime \prime N ~ 71 ³ 6 ' 23.5 ” W, ~ 449 m s n m . ~ 09 . I V .2009, ~ G . ~ d e ~ l o s ~ S a n t o s . ~}$

\section{Megalostrata raptor (L. Koch, 1866)}

Material examinado: $1 \widehat{\jmath}$, CGA. San Cristóbal, República Dominicana. 11.VII.1978, M. Olvisa.

Familia Gnaphosidae

Camillina Berland, 1919

Material examinado: 1q, MNHNSD 09.1261. Las Salinas, Las Calderas, Baní, prov. Peravia, República Dominicana. 02.XII.2007, A. Sánchez.

\section{Zelotes Gistel, 1848}

Material examinado: $3 \propto q+1$ juv., MNHNSD 09.1233. Boca de Cachón, La Descubierta, prov. Independencia, República Dominicana. 18³3'27.9”N 7149'59.5”W, 3mbnm. 06.IX.2009, G. de los Santos. 1 9 , MNHNSD 09.1232. La Furnia, Las Clavellinas, Los Ríos, prov. Bahoruco, República Dominicana. 18³0’37.3”N 71³4'14.8”W, 22mbnm. 08.I.2010, G. de los Santos. 1 , MNHNSD 09.1234. Sabana Quéliz, Constanza, prov. La Vega, Parque Nacional Valle Nuevo, República Dominicana. UTM 19Q 327676m.E 2074940m.N, 2,286msnm. 24.VII.2010, S. Carrero, G. de los Santos, sobre Danthonia domingensis.

Familia Philodromidae

Tibellus Simon, 1875

Material examinado: 1q, MNHNSD 09.1250. La Zurza, Duvergé, prov. Independencia, República Dominicana. 18²4'25.1'N 71³4’25.4”W, 28 mbnm. 05.IX.2009, G. de los Santos.

Familia Thomisidae

Xysticus pellax O. P.-Cambridge, 1894

Material examinado: 1 , $2 \widehat{\diamond}$ MNHNSD 09.1249. La Pirámide, Constanza, prov. La Vega, Parque Nacional Valle Nuevo, República Dominicana. UTM 19Q 331313m.E 2069156m.N,

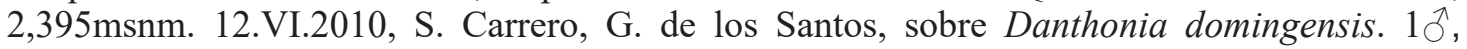
1 inm., MNHNSD 09.1253. La Pirámide, Constanza, prov. La Vega, Parque Nacional Valle Nuevo, República Dominicana. UTM 19Q 331340m.E 2069149m.N, 2,395msnm. 19.I.2011, S. Carrero, G. de los Santos, sobre Danthonia domingensis. 16 juv. MNHNSD 09.1254. La 
Lechuguilla, Constanza, prov. La Vega, Parque Nacional Valle Nuevo, República Dominicana. UTM 19Q 327676m.E 2074940m.N, 2,286msnm. 24.VIII.2010, S. Carrero, G. de los Santos, sobre Danthonia domingensis. 2ð̄̄ 1 juv., MNHNSD 09.1255. Sabana Quéliz, Constanza, prov. La Vega, Parque Nacional Valle Nuevo, República Dominicana. UTM 19Q 327724m.E 2074956m.N, 2,290msnm. 25.VIII.2010, S. Carrero, G. de los Santos, sobre Danthonia domingensis. 1 ㅇ, $2 \hat{\jmath}, 6$ inm. MNHNSD 09.1256. Sabana Quéliz, Constanza, prov. La Vega, Parque Nacional Valle Nuevo, República Dominicana. UTM 19Q 327568m.E 2075027m.N, 2,228msnm. 15.I.2011, S. Carrero, G. de los Santos, sobre Danthonia domingensis. 1, $1 \delta^{\lambda}$, 10 juv., MNHNSD 09.1257. Sabana Quéliz, Constanza, prov. La Vega, Parque Nacional Valle Nuevo, República Dominicana. UTM 19Q 327724m.E 2074956m.N, 2,290msnm. 14.I.2011, S. Carrero, G. de los Santos, sobre Danthonia domingensis. 1 \& MNHNSD 09.1258. La Lechuguilla, Constanza, prov. La Vega, Parque Nacional Valle Nuevo, República Dominicana. UTM 19Q 331501m.E 2070693m.N, 2,412msnm. 26.VIII.2010, S. Carrero, G. de los Santos, sobre Danthonia domingensis.

\section{AMPLIACIÓN DE DISTRIBUCIÓN}

Familia Ochyroceratidae

Ochyrocera cachote Hormiga, Álvarez-Padilla et Benjamin, 2007

Material examinado: $1+10 \hat{\jmath}$, MNHNSD 09.1245. La Lechuguilla, Constanza, prov. La Vega, Parque Nacional Valle Nuevo, República Dominicana. UTM 19Q 331530m.E 2070703m.N, 2,362msnm. 16.I.2011, S. Carrero, G. de los Santos, sobre Danthonia domingensis. $2 \hat{\jmath} \widehat{\jmath}$, MNHNSD 09.1246. La Pirámide, Constanza, prov. La Vega, Parque Nacional Valle Nuevo, República Dominicana. UTM 19Q 331340m.E 2069149m.N, 2,395msnm. 19.I.2011, S. Carrero, G. de los Santos, sobre Danthonia domingensis. 1 juv., MNHNSD 09.1244. Sabana Quéliz, Constanza, prov. La Vega, Parque Nacional Valle Nuevo, República Dominicana. UTM 19Q 327724m.E 2074956m.N, 2,290msnm. 25.VIII.2010, S. Carrero, G. de los Santos, sobre Danthonia domingensis. 1§, MNHNSD 09.1248. La Lechuguilla, Constanza, prov. La Vega, Parque Nacional Valle Nuevo, República Dominicana. UTM 19Q 331501m.E 2070693m.N,

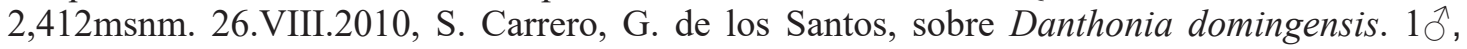
MNHNSD 09.1247. La Pirámide, Constanza, prov. La Vega, Parque Nacional Valle Nuevo, República Dominicana. UTM 19Q 331313m.E 2069156m.N, 2,395msnm. 12.VI.2010, S. Carrero, G. de los Santos, sobre Danthonia domingensis.

Registros anteriores: Bosque nublado rodeado de vegetación en crecimiento secundario, Reserva Natural Cachote, Paraíso, prov. Barahona, República Dominicana. $18^{\circ} 05^{\prime} 54.8^{\prime} \mathrm{N}$ 71¹1'22.0'W, 1,220 msnm, 6-9.IV.2005, G. Hormiga, F. Álvarez-Padilla, S. P. Benjamín, 4

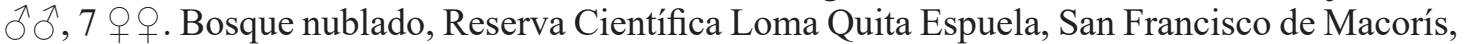
prov. Duarte, República Dominicana. 19²1'00.6'N 7008'31.0"W, 942 msnm, 19-21.IV.2005, F. Álvarez-Padilla, S. P. Benjamín, 2 ふ̄ô, 3 q + (Hormiga et al., 2007).

Familia Ctenidae

Trujillina spinipes Bryant, 1948

Material examinado: 1 , MNHNSD 09.1230. El Manaclar, La Montería, Baní, prov. Peravia, República Dominicana. UTM 19Q 352357m.E 2035657m.N, 1,358msnm. 26.IX.2009, G. de los Santos.

Registros anteriores: $29 q$ (Holotipo y Paratipo), Loma Rucilla, Cordillera Central, República

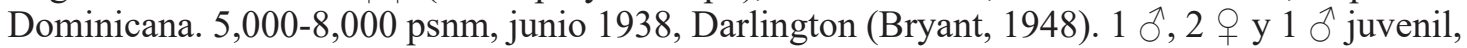
$11 \mathrm{Kms}$. del entronque de Abanico, Casabito, Cordillera Central, provincia Monseñor Nouel, 12-I- 1991, Luís R. Hernández (Alayón, 1995). 
Familia Salticidae

Cobanus sp.

Material examinado: 1ð̄, MNHNSD 09.1229. El Manaclar, La Montería, Baní, prov. Peravia, República Dominicana; transepto loma Los Guayuyos-Segundo Río. UTM 19Q 354040m.E 2036965m.N, 1,278msnm. 29.VIII.2009, G. de los Santos.

Registros anteriores: (como Cobanus cambridgei Bryant, 1943) 1ð, Cordillera Central, Constanza, República Dominicana. 3,000-4,000 psnm, agosto 1938, Darlington. 19, $2 \widehat{\jmath}$, San José de las Matas, República Dominicana. 1,500 psnm, junio 1938, Darlington. Estos registros corresponden a Cobanus cambridgei Bryant, 1943 (Bryant, 1943).

\section{Familia Liocranidae}

Lausus pulchellus Bryant, 1948

Material examinado: 1 juv., MNHNSD 09.1242. Sabana Quéliz, Constanza, prov. La Vega, Parque Nacional Valle Nuevo, República Dominicana. UTM 19Q 327724m.E 2074956m.N, 2,290msnm. 25.VIII.2010, S. Carrero, G. de los Santos, sobre Danthonia domingensis. 19, MNHNSD 09.1243. Sabana Quéliz, Constanza, prov. La Vega, Parque Nacional Valle Nuevo, República Dominicana. UTM 19Q 327676m.E 2074940m.N, 2,286msnm. 24.VIII.2010, S. Carrero, G. de los Santos, sobre Danthonia domingensis. 19, MNHNSD 09.1241. La Pirámide, Constanza, prov. La Vega, Parque Nacional Valle Nuevo, República Dominicana. UTM 19Q 331313m.E 2069156m.N, 2,395msnm. 12.VI.2010, S. Carrero, G. de los Santos, sobre Danthonia domingensis.

Familia Araneidae

Eustala bisetosa Bryant, 1948

Material examinado: 1q, MNHNSD 09.1190. Loma Los Pinos, El Manaclar, La Montería, Baní, prov. Peravia, República Dominicana. UTM 19Q 352357m.E 2035657m.N, 1,358 msnm, 26.IX.2009, G. de los Santos. 19, MNHNSD 09.1191. Vertiente SO loma Los Guayuyos, El Manaclar, La Montería, Baní, prov. Peravia, República Dominicana. UTM 19Q 353421m.E 2036933m.N, 1,377 msnm, 25.IX.2009, G. de los Santos. 4q $q$, MNHNSD 09.1218. Alto de la Rosa, Sabaneta, prov. San Juan, República Dominicana. Parque Nacional José del Carmen Ramírez. 1902'21'”N 71 13’20”W, 1,773msnm. 22-23.XI.2009, R. Ortiz, G. de los Santos.

Registros anteriores: $1 \widehat{\jmath}$, Pié de las colinas de la Cordillera Central, al sur de Santiago, República Dominicana. 1,000-3,000 psnm, junio 1938, Darlington. 1ô, 1 , , Kenskoff, Haití. 4,500-5,500 psnm, 02.IX.1934, Darlington. 5우, Kenskoff, Haití. 3,500 psnm, 03.V.1935, Roys (Bryant, 1945).

\section{Larinia minor Bryant, 1945}

Material examinado: 1 q, MNHNSD 09.209. Playa Los Patos, Los Patos, Paraíso, prov. Barahona, República Dominicana. 31.VIII.1976, J. A. Ottenwalder. 3우, 10̄, MNHNSD 09.1220. La Furnia, Las Clavellinas, Los Ríos, prov. Bahoruco, República Dominicana. 18³0’37.3'N

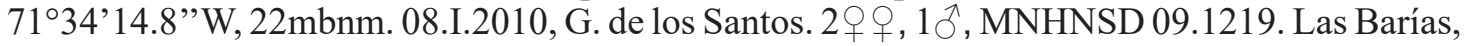
La Descubierta, prov. Independencia, República Dominicana. 18³3'43.8'” $71^{\circ} 43^{\prime} 31.4$ ' W, 21mbnm. 09.IV.2010, G. de los Santos.

Registros anteriores: 1q, Puerto Príncipe, Haití. Julio 1941, Audant (Bryant, 1945; Harrod et al. 1991). 


\section{DISCUSIÓN}

Estos nuevos registros aumentan el número de especies conocidas para La Hispaniola a 366 (en la fauna actual), agrupadas en 48 familias. Con la excepción de Orchestina Simon, 1882, Pholcomma Thorell, 1869 y Tapinopa bilineata Banks, 1893, los demás nuevos registros poseen representantes en las Antillas. El género Orchestina Simon, 1882, ya poseía tres registros en la fauna fósil de La Hispaniola (Perez-Gelabert, 2008). Del género Pholcomma Thorell, 1869 se conocen solo cinco especies en todo el complejo del continente Americano (i.e. EUA, Canadá, Argentina y Brasil); mientras que Tapinopa bilineata Banks, 1893, se conocía solamente de los Estados Unidos de Norteamérica (Platnick, 2012). Estos tres registros también constituyen los primeros que se hacen de estos géneros en la fauna actual del Caribe (Fig. 1).

De acuerdo con Avila Calvo (2000) y Platnick (2012), previo al nuevo registro del género Neohannia Mello-Leitão, 1917, solo se ha descrito una especie de la famalia Hahniidae en las Antillas, en la isla de Puerto Rico; y hay otro registro de Cuba.

El género Mazax O. P.-Cambridge, 1898, es americano y cuenta con seis especies descritas, encontrándose una especie en Jamaica y otra en las Antillas Menores.

Los géneros Camillina Berland, 1919 y Zelotes Gistel, 1848 (familia Gnaphosidae) en las Antillas cuentan con ocho y tres especies, respectivamente (Platnick y Shadab, 1982, 1983; Platnick, 2012). En esta área, Camillina Berland posee representantes en las islas de Puerto Rico (2 especies), Cuba (2), Jamaica (2), Curazao (1) y una especie de amplia distribución en el Caribe; mientras que Zelotes Gistel, cuenta con una especie en Cuba y dos en Jamaica. Debido a la conocida relación biogeográfica entre estas islas, era de esperarse que tarde o temprano aparecieran representantes de estos géneros en La Hispaniola; que en el caso de Zelotes Gistel pudieran tratarse de más de una especie debido a las diferencias extremas entre los ambientes de colecta.

Por otra parte, de los géneros Tibellus Simon, 1875 y Xysticus C. L. Koch, 1835, se conocían registros en Cuba, ambos con una especie. Este es el primer registro de Xysticus pellax O. P.Cambridge fuera de Norteamérica.

En cuanto a las ampliaciones de distribución de las especies endémicas, el araneido Larinia minor Bryant solo se conocía de la localidad tipo, en Haití (Bryant, 1945; Harrod et al., 1991), siendo este el primer registro de esta especie para la República Dominicana. De acuerdo con las nuevas localidades de colecta esta especie parece encontrarse en la parte suroeste de la isla (Fig. 2). Eustala bisetosa Bryant se conocía de dos localidades (una en Haití, una en República Dominicana) y en este trabajo se amplían a cinco. Todas las localidades conocidas en la República Dominicana se encuentran en la Cordillera Central.

La localidad de colecta del género Cobanus representa una ampliación en la distribución de este género, del cual Cobanus cambridgei Bryant es el único representante conocido hasta el momento. Trujillina spinipes Bryant, descrita hace más de 60 años, se conocía solamente de dos localidades.

Por otro lado, Ochyrocera cachote Hormiga, Álvarez-Padilla et Benjamin se conocía de bosques nublados en dos áreas protegidas de la República Dominicana (Hormiga et al., 2007) y ahora se registra por primera vez en bosques de pinos (Pinus occidentalis Swartz), asociada a la sabana de pajón (Danthonia domingensis) en el Parque Nacional Valle Nuevo. 


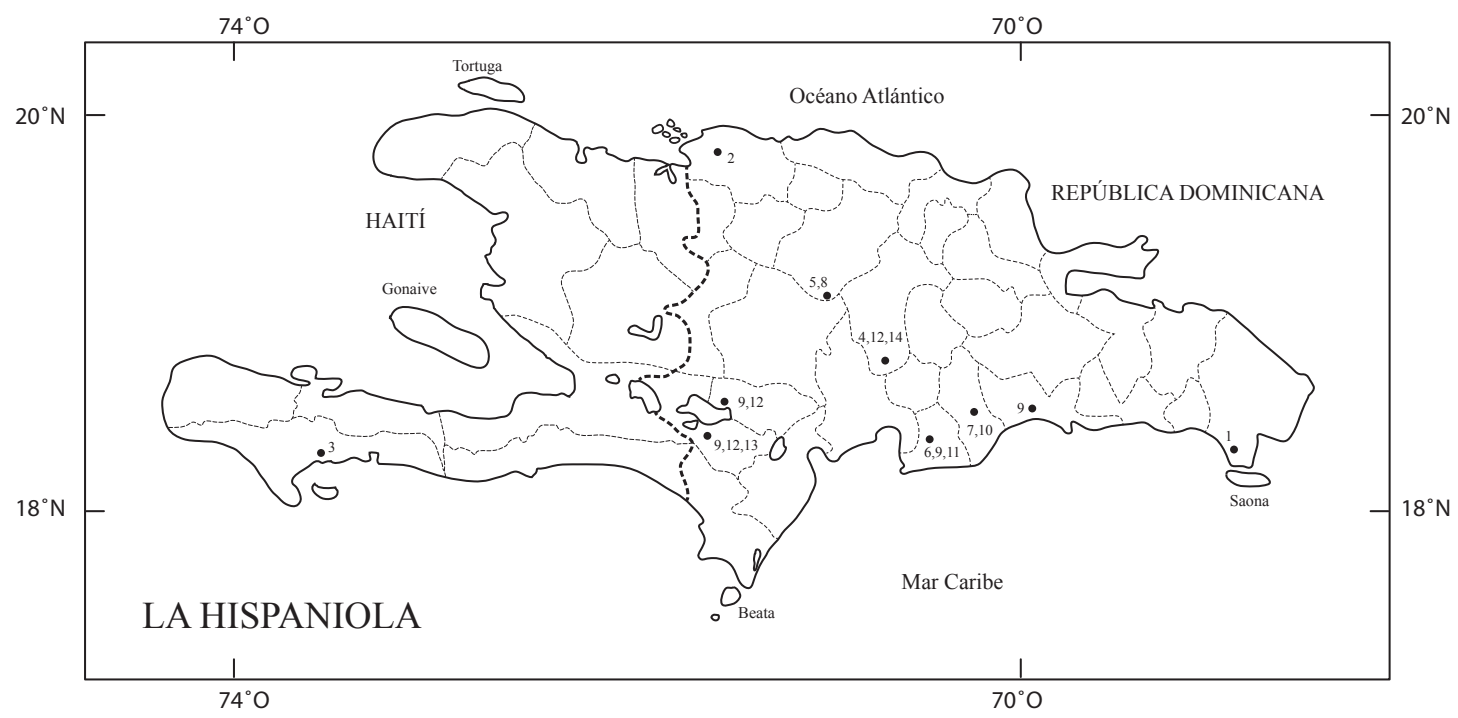

Figura 1. Localidades de colecta de los nuevos registros. Orchestina sp. (1), Mimetus notius Chamberlin (2), Pholcomma sp. (3), Tapinopa bilineata Banks (4), Leucauge moerens (O. P.-Cambridge) (5), Kapogea alayoi (Archer) (6), Ctenus ottleyi (Petrunkevitch) (7), Neohannia sp. (8), Mazax sp. (9), Megalostrata raptor (L. Koch) (10), Camillina sp. (11), Zelotes sp. (12), Tibellus sp. (13) y Xysticus pellax O. P.-Cambridge (14).

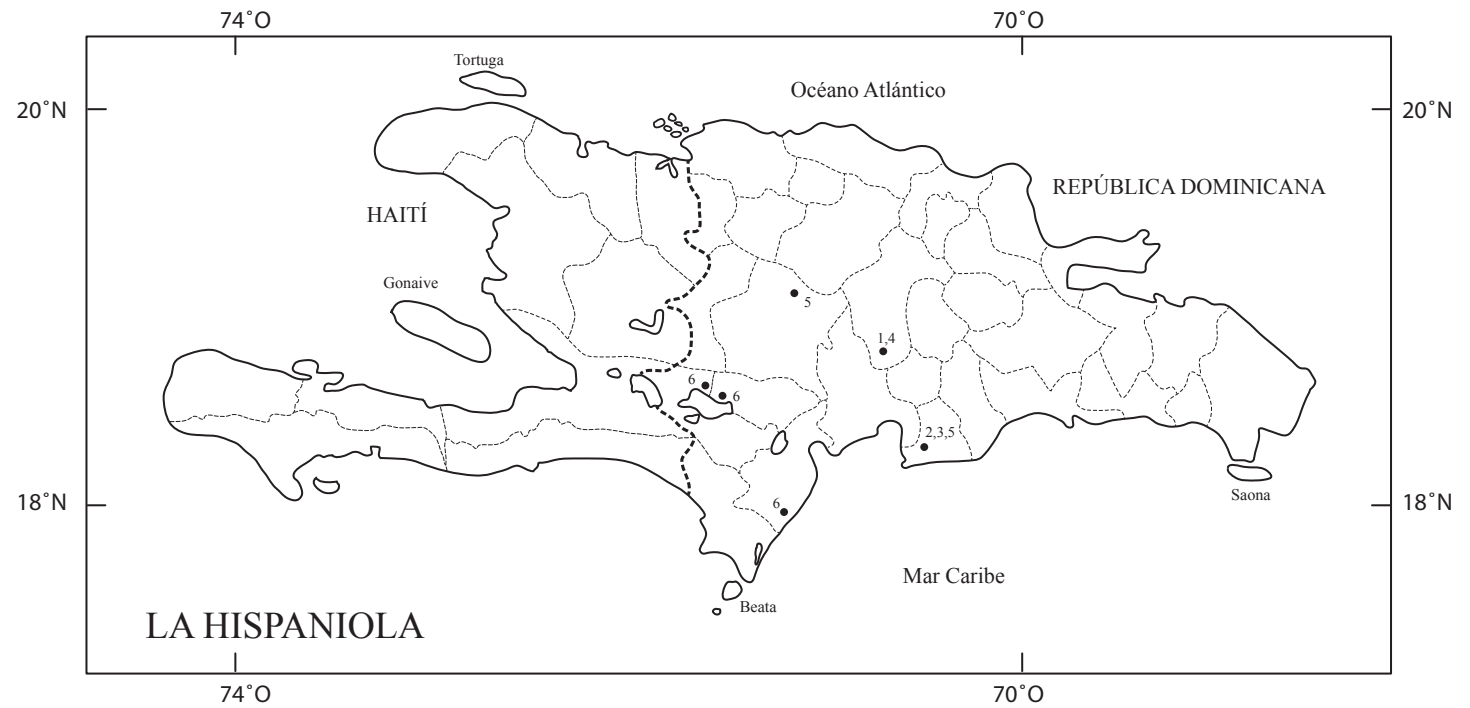

Figura 2. Ampliaciones de rango de distribución para las especies: Ochyrocera cachote Hormiga, Álvarez-Padilla \& Benjamin (1), Trujillina spinipes Bryant (2) Cobanus sp. (3), Lausus pulchellus Bryant (4), Eustala bisetosa Bryant (5) y Larinia minor Bryant (6).

\section{AGRADECIMIENTOS}

Se agradece a Ruth Bastardo (IIBZ, UASD), investigadora principal del proyecto "Reconocimiento de los Recursos Naturales del Área Importante para la Conservación de las Aves Honduras (AICA Honduras) y su entorno, Provincia Peravia, República Dominicana"; a Gladys Rosado (CIBIMA, UASD), investigadora principal del proyecto "Caracterización ambiental de los humedales: La Azufrada, Las Marías, Las Barías, Boca de Cachón y La Zurza, que bordean el Lago Enriquillo, provincia Bahoruco, República Dominicana", y a Celeste Mir (MNHNSD), investigadora principal del proyecto "Ecología de la fauna asociada al suelo en la 
Sabana de Pajón del Parque Nacional Juan Bautista Pérez Rancier (Valle Nuevo) y su respuesta a los impactos de la agricultura y el fuego" por incluir a las arañas entre sus grupos de interés y hacer posible las expediciones que permitieron la colecta de los ejemplares. Estos trabajos fueron posibles gracias al Fondo Nacional de Innovación y Desarrollo Científico y Tecnológico (FONDOCYT) del Ministerio de Educación Superior Ciencia y Tecnología (MESCyT) de la República Dominicana. Alexander Sánchez-Ruiz (BIOECO) hizo importantes correcciones y sugerencias que mejoraron el manuscrito.

\section{LITERATURA CITADA}

Alayón, G. 1995. Descripción del macho de Trujillina spinipes Bryant (Araneae: Ctenidae). Poeyana, 452: 1-8.

Alayón, G. 2011. Apuntes sobre la familia Araneidae, con la descripción de dos especies nuevas de Wagneriana (Araneae: Araneidae) de Cuba y República Dominicana. Solenodon 9: 2028.

Alayón, G. y G. de los Santos. 2009. Primer reporte de la familia Cyrtaucheniidae (Araneae: Mygalomorphae) en la fauna actual de La Hispaniola. Novitates Caribaea 2: 45-46.

Avila Calvo, Arturo F. 2000. Primer registro de la familia Hahniidae (Arachnida: Araneae) para Cuba. Avicennia, 12/13: 135-136.

Bryant, E. B. 1943. The salticid spiders of Hispaniola. Bull. Mus. Comp. Zool. Harv. 92: 445529.

Bryant, E. B. 1945. The Argiopidae of Hispaniola. Bull. Mus. Comp. Zool. Harv. 95: 357-422.

Bryant, E. B. 1948. The spiders of Hispaniola. Bull. Mus. Comp. Zool. Harv. 100: 331-459.

De los Santos, G. y S. Carrero. 2011. La familia Araneidae (Arachnida: Araneae) en la colección del Museo Nacional de Historia Natural de Santo Domingo y dos nuevos registros para La Hispaniola. Novitates Caribaea 4: 54-64.

Harrod, J. C., H. W. Levi y L. B. Leibensperger. 1991. The Neotropical orbweavers of the genus Larinia (Araneae: Araneidae). Psyche, Camb. 97: 241-265.

Hormiga, G., Alvarez-Padilla, F. y Benjamin, S. P. 2007. First records of extant Hispaniolan spiders of the families Mysmenidae, Symphytognathidae, and Ochyroceratidae (Araneae), including a new species of Ochyrocera. American Museum Novitates, 3577, 1-21.

Huber, B. A. y J. J. Astrin. 2009. Increased sampling blurs morphological and molecular species limits: revision of the Hispaniolan endemic spider genus Tainonia (Araneae: Pholcidae). Invertebr. Syst. 23: 281-300.

Huber, B. A., N. Fischer y J. J. Astrin. 2010. High level of endemism in Haiti's last remaining forests: a revision of Modisimus (Araneae: Pholcidae) on Hispaniola, using morphology and molecules. Zool. J. Linn. Soc. 158: 244-299.

Perez-Gelabert, D. E. 2008. Arthropods of Hispaniola (Dominican Republic and Haiti): A checklist and bibliography. Zootaxa 1831: 1-530. 
Platnick, N. I. 2012. The world spider catalog, version 12.5. American Museum of Natural History, online at http://research.amnh.org/iz/spiders/catalog.

Platnick, N. I. y M. U. Shadab. 1982. A revision of the American spiders of the genus Camillina (Araneae, Gnaphosidae). American Museum Novitates 2748: 1-38.

Platnick, N. I. y M. U. Shadab. 1983. A revision of the American spiders of the genus Zelotes (Araneae, Gnaphosidae). Bull. Am. Mus. Nat. Hist. 174: 97-192.

Sánchez-Ruiz, A. 2009. Nuevos registros y ampliaciones de ámbito geográfico para las arañas (Arachnida: Araneae) de La Hispaniola, Antillas Mayores. Novitates Caribaea 2: 23-29.

Sánchez-Ruiz, A., N. I. Platnick y N. Dupérré. 2010. A new genus of the spider family Caponiidae (Araneae, Haplogynae) from the West Indies. American Museum Novitates 3705: 1-44.

[Recibido: 16 de mayo, 2012. Aceptado para publicación: 04 de junio, 2012] 